\title{
Komplementärmedizin in der Grundversicherung
}

\section{Gisela Etter}

Dr. med., Allgemeine Innere Medizin, Mitglied FMH, Homöopathie (SVHA)

\author{
Die obligatorische Krankenpflegeversicherung wird die ärztlichen komplementär- \\ medizinischen Leistungen weiterhin übernehmen, und zwar unbefristet.
}

An seiner Sitzung vom 16. Juni 2017 hat der Bundesrat die neuen Verordnungsbestimmungen genehmigt, welche die komplementärmedizinischen ärztlichen Leistungen den anderen von der OKP vergüteten medizinischen Fachrichtungen gleichstellen. Die neuen Regelungen traten per 1. August 2017 in Kraft.

Im Mai 2009 haben Volk und Stände den neuen Verfassungsartikel zur Berücksichtigung der Komplementärmedizin deutlich angenommen. Seit 2012 vergütet die OKP die ärztlichen Leistungen der anthroposophischen Medizin, der Arzneimitteltherapie der Traditionellen Chinesischen Medizin (TCM), der klassischen Homöopathie und der Phytotherapie. Diese Kostenübernahme war jedoch bis Ende 2017 befristet.

\section{Der Statuswechsel der ärztlichen komplementärmedizinischen Leistungen hat keine finanziellen Auswirkungen.}

Die Akupunktur, die bereits unbefristet von der OKP vergütet war, wurde neben den vier provisorisch vergüteten Fachrichtungen ebenfalls in diese Gleichstellung einbezogen.

Der Statuswechsel der ärztlichen komplementärmedizinischen Leistungen hat keine finanziellen Auswirkungen.

Die UNION Schweizerischer komplementärmedizinischer Ärzteorganisationen begrüsst diesen Entscheid des Bundesrates.

Der neue Lernzielkatalog «PROFILES» (= Principal Relevant Objectives and Framework for Integrated Learning and Education in Switzerland) wurde am 15. März 2017 definitiv durch die Schweizerische Medizinische Interfakultätskommission (SMIFK/CIMS) genehmigt. Auch hier wird die Komplementärmedizin, wie im Medizinalberufegesetz (MedBG) aus dem Jahr 2013 gefordert, klar berücksichtigt. Nun bedarf es jedoch der korrekten Umsetzung an den einzelnen Universitäten, damit dem Volkswillen von 2009 Genüge getan wird!
Die UNION Schweizerischer komplementärmedizinischer Ärzteorganisationen vereinigt als Dachverband über 1000 Ärztinnen und Ärzte, welche zusätzlich zu ihrer konventionellen Facharztausbildung eine Weiterbildung gemäss Fähigkeitsprogrammen in Phytotherapie, klassischer Homöopathie, anthroposophisch erweiterter Medizin oder Akupunktur - Arzneimitteltherapie der Traditionellen Chinesischen Medizin (TCM) des Schweizerischen Instituts für Weiter- und Fortbildung (SIWF) innehaben.

Die UNION entstand 1996 aus der "Arbeitsgruppe Komplementärmedizin» heraus und dient den Behörden und der FMH seither als einheitlicher Gesprächspartner in allen Fragen zur ärztlichen Komplementärmedizin.

Dass sich die UNION auch für Qualität einsetzt, hat sie gezeigt, indem sie 2016 mit zahlreichen anderen Ärzteorganisationen die Qualitäts-Charta der FMH unterzeichnet hat.

Die vier in der UNION vereinten Ärzteorganisationen bieten interessante und flexible Curricula zu den entsprechenden Fähigkeitsausweisen. Diese werden von den einzelnen Fachgesellschaften verwaltet, sind vom Schweizerischen Institut für Weiter- und Fortbildung (SIWF) anerkannt und berechtigen zur Abrechnung der speziellen Tarifpositionen im TARMED. Weitere Informationen finden sich auf der Website des SIWF oder der einzelnen Fachgesellschaften.

\section{Vorstand}

Präsidentin: Dr. med. Gisela Etter

Vizepräsidentin: Dr. med. Anita Meyer

Kassier: Dr. med. Roger Eltbogen

Vorstandsmitglieder: Dres. med. Brigitte Ausfeld-Hafter,

Franziska Bläuer, Roland Koller 